\title{
Centro de intervención en apoyo a alumnos de carreras de ingeniería
}

\section{Center of intervention in support to students of engineering careers}

\author{
ALANÍS, Reynaldo†*, URUETA, Daniel E., ALANÍS, José A. y BRITO, Suzuki \\ Universidad Politécnica del Estado de Guerrero, Comunidad de Puente Campuzano Carretera Federal Iguala - Taxco \\ K.M. 105 C.P. 40321, Municipio de Taxco de Alarcón, Gro. 3rd: Facultad de Arte y Diseño, Universidad Autónoma de \\ México.
}

ID $1^{\mathrm{er}}$ Autor: Reynaldo, Alanís / ORC ID: 0000-0001-5397-7016

ID $1^{\mathrm{er}}$ Coautor: Daniel E., Urueta / ORC ID: 0000-0002-8741-6978

ID $2^{\text {do }}$ Coautor: José A., Alanís / ORC ID: 0000-0003-3337-2380

ID $3^{\text {er }}$ Coautor: Suzuki, Brito

DOI: $10.35429 / J O T E .2020 .11 .4 .17 .22$

Recibido Enero 15, 2020; Aceptado Marzo 31, 2020

\begin{abstract}
Resumen
Los objetivos de este trabajo es presentar un modelo de flujo de trabajo; tres pruebas y dos cuestionarios para diagnosticar desempeño académico; el uso de metodologías estadísticas para el análisis de los datos obtenidos, así como los resultados preliminares del modelo y de los cuestionarios. La metodología consiste en la coordinación entre docentes en el rol de tutor y aplicar las pruebas y cuestionarios a los alumnos bajo su responsabilidad para posteriormente realizar un análisis estadístico de los datos y en base a ello elaborar un plan de trabajo de intervención a nivel individual y colectivo. La contribución fundamental de este trabajo es buscar de manera objetiva apoyar a los alumnos en su desarrollo académico. Dentro de las responsabilidades y actividades que un docente tiene en las universidades es ser tutor de un grupo del programa académico al que pertenece. Los alumnos bajo su responsabilidad tienen una serie de situaciones que influyen o suponemos que influyen en su desempeño académico. Uno como tutor se puede preguntar si las unidades de aprendizaje de las asignaturas que cursan apoyan en dicho desempeño, si existen factores internos y externos, así como situaciones de riesgo que lo afecten. Este trabajo está orientado a apoyar a los docentes en su actividad tutora y en las asignaturas transversales del programa académico, así como a los alumnos.
\end{abstract}

Educación, Desempeño académico, Factores en el aprendizaje

\begin{abstract}
The objectives of this work are to present a workflow model; three tests and two questionnaires for diagnosis; statistical methodologies for the analysis of the data obtained, as well as the preliminary results of the model and the questionnaires. The methodology consists of the coordination between teachers in the role of tutor and the application to students under their responsibility to subsequently perform a statistical analysis of the data and based on this, develop an intervention work plan at the individual and collective level. The fundamental contribution of this work is to objectively seek to support students in their academic development. Among the responsibilities and activities that a teacher has in universities is to be a tutor of a group of the academic program to which he belongs. Students under responsibility have a series of situations that influence or assume that they influence their academic performance. One as a tutor can ask if the learning units of the subjects, they take support this performance, if there are internal and external factors, as well as risk situations that affect it. This work is aimed at supporting teachers in their tutoring activity and in the transverse subjects of the academic program, as well as students.
\end{abstract}

Education, Academic performance, Factors in learning

Citación: ALANÍS, Reynaldo, URUETA, Daniel E., ALANÍS, José A. y BRITO, Suzuki. Centro de intervención en apoyo a alumnos de carreras de ingeniería. Revista de Educación Técnica. 2020. 4-7:17-22.

\footnotetext{
*Correspondencia al Autor (Correo Electrónico: ralanis2513@gmail.com)
}

$\dagger$ Investigador contribuyendo como primer autor. 


\section{Introducción}

El desempeño académico de los alumnos en los diferentes programas educativos (PE) de ingeniería dependen de diversos factores tanto académicos como sociales y personales. Los alumnos cursan una serie de asignaturas del programa académico y se puede uno preguntar si las unidades de aprendizaje (UA) de dichas asignaturas están aportando a las competencias que se espera desarrollen, dado que el enfoque de aprendizaje que se sigue en la universidad es Basado en Competencias (Argudín, (2011)).

Los alumnos pueden modificar su desempeño académico positiva o negativamente debido a situaciones distintas a las UA de las asignaturas (García, \& Galán (1991)). El docente puede preguntarse si dichas situaciones están afectando al desempeño del alumno, cuáles podrían ser y que tanto. Bajo ciertas circunstancias ya sean académicas, de actitud, de comportamiento de algún alumno o del grupo de alumnos en algún grupo en particular, el docente puede acudir al tutor y mencionar situaciones "anómalas" para ayudar y mejorar su trabajo y el de sus alumnos y él, como tutor que es, también podría ser abordado por las mismas causas. Existen diferentes instrumentos que pueden apoyar a los docentes en su labor como responsables de alguna asignatura en particular o en su rol como tutor o como colaborador con los tutores de otros grupos (Kaplan, R. M., \& Saccuzzo, D. P. 2006).

Por otra parte, una vez que se tiene el diagnóstico por alumno y por grupo los tutores y los docentes del grupo tutorado pueden elaborar, en coordinación con los demás departamentos de la universidad (enfermería, deportes, cultura, legal, jefatura, secretaría académica, rectoría), planes de intervención que apoyen a los alumnos en forma individual o en grupo. El diagnóstico debe fundamentarse en metodologías y herramientas formales, tales como Estadística Descriptiva e Inferencial, Análisis de Regresión Lineal (Chávez, Pérez, Alanís (2019)). Hay alumnos que han desertado de la carrera o se les dio de baja y se pudo evitar con un poco de atención o mejorando el desempeño del grupo con actividades individuales o grupales.
Por lo anterior, el trabajo del docente tanto en la asignatura como en su rol de tutor se podría dividir en: evaluación, diagnóstico e intervención. Surgen las siguientes interrogantes ¿qué se puede usar para la evaluación? ¿cómo realizar el diagnóstico lo más objetivo posible? ¿cómo medir el avance del alumno independientemente de los instrumentos de evaluación sugeridos en los manuales de asignatura? ¿cómo medir objetivamente o cuantitativamente si influye en el alumno o grupo la relación con los docentes o sus compañeros, las instalaciones o la selección de la carrera? ¿cómo ayudar al grupo $\mathrm{y}$ al individuo a progresar en su aprendizaje? ¿cómo medir el avance en las competencias correspondientes de cada ciclo de formación más allá de lo que evalúan las evidencias sugeridas por los manuales de asignatura correspondientes? Se puede utilizar para determinar el avance cognitivo de los alumnos tests que evalúan habilidades numéricas, razonamiento abstracto y mecánico. En el caso de factores internos o externos, así como situaciones de riesgo que afectan o pueden afectar al alumno y al grupo se pueden elaborar cuestionarios para ese propósito. Con los resultados de las pruebas y los cuestionarios, se puede realizar el análisis estadístico y en colaboración con profesionales en áreas de la salud, deporte y cultura, se puede proponer una estrategia de intervención tanto a nivel individual como de grupo.

\section{Objetivos}

- Proponer un modelo de flujo de trabajo para un Centro de Intervención en Apoyo a Alumnos de las Carreras de Ingeniería (CIAACI).

- Seleccionar tests y cuestionarios para aplicar a los alumnos para el diagnóstico.

- Seleccionar las metodologías estadísticas para el análisis de las pruebas y cuestionarios.

- Aplicar las pruebas y cuestionarios y presentar resultados preliminares.

- Determinar el grado de dependencia de parámetros sociales o económicos con el desempeño académico del alumno.

- $\quad$ Proponer posibles intervenciones. 


\section{Metodología}

\section{Modelo de flujo de trabajo}

El flujo de trabajo, figura 1, inicia con una reunión con los docentes (son tutores también) de las asignaturas que se impartirán en el ciclo escolar. En el caso particular de la Universidad Politécnica del Estado de Guerrero (UPEGro), son cuatrimestres. Cada docente revisará el manual correspondiente a la asignatura que impartirá identificando las UA a cubrir. En esa reunión, los tutores podrán planear y realizar el programa de tutoría que solicita la Dirección de Carrera y la Secretaría Académica para ese cuatrimestre. Se explicarían las pruebas y cuestionarios a los tutores que los desconocen. En el plan, se programaría la aplicación de las pruebas y cuestionarios correspondientes.

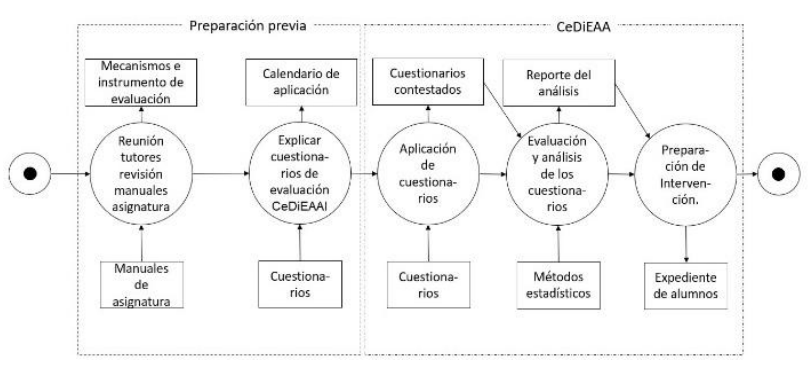

Figura 1 Modelo del flujo de trabajo del CIAACI

\section{Test y cuestionarios}

Se identificaron y se propone el uso de instrumentos que miden Habilidad Numérica (HN), Razonamiento Mecánico (RM) y Razonamiento Abstracto (RA). En la figura 2 se muestra un esquema de la relación que existe entre el alumno, las asignaturas, los manuales de asignatura, las UA, los cuatrimestres, los ciclos de formación, así como los instrumentos de evaluación.

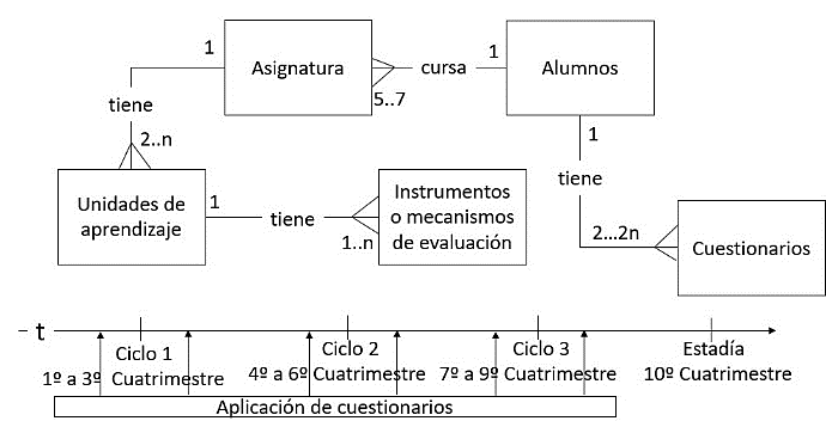

Figura 2 Esquema de relación del alumno
Las pruebas son: Test de Aptitud Diferencial para universidades que mide: Habilidad Numérica, Razonamiento Mecánico y Razonamiento Abstracto. Ellos permiten evaluar aptitudes múltiples. Diseñado para alumnos de nivel medio y de nivel superior. Se publicaron en 1947 y su confiabilidad es entre 0.85 a 0.93 (Kaplan, R. M., \& Saccuzzo, D. P. 2006).

Se seleccionó el que evalúa la HN pues mide principios básicos en matemáticas tales como: inducción y deducción para solucionar situaciones en el que se debe utilizar el concepto de número. Por otro lado, el test RM, permite evaluar habilidades relacionadas con entender principios mecánicos básicos del funcionamiento de máquinas y herramientas, así mismo evalúa la capacidad en el manejo de objetos y la comprensión de mecanismos. RM es ideal pues evalúa el avance en el razonamiento de los alumnos de los $\mathrm{PE}$ en ciencia e ingeniería.

El test RA es el apropiado ya que los alumnos de los PE en ciencias e ingeniería deben desarrollar dicha habilidad. Evalúa: capacidad de observación, la forma lógica de organizar lo observado para obtener conclusiones basándose en datos concretos usando lógica deductiva. Los estudiantes de los $\mathrm{PE}$ en ciencias e ingeniería deben desarrollar esta habilidad ya que en ambos se realizan modelos matemáticos, pasando de la realidad a la abstracción y viceversa.

El test RA incluye: secuencia de imágenes tales como series de números, monedas, naipes, letras, fichas de dominó o figuras. Cabe mencionar que los instrumentos HN, RM y RA lo aplicaron los autores de la Universidad Autónoma de Nuevo León en el 2018 a estudiantes de ciencias (Chávez Valdez 2019). Los instrumentos HN, RM y RA se propone aplicarlos durante la carrera. En el caso de las carreras que se imparten en la UPEGro sería al inicio y final de cada ciclo de formación, el cual consta de tres cuatrimestres y hay tres ciclos de formación como se mostró en la figura 2. 
Los cuestionarios que se eligieron los desarrollaron los coautores de este trabajo. Navarro J. Andrés desarrolló un cuestionario que consta de 24 preguntas agrupadas en tres secciones: datos generales del alumno, historial académico y los datos clasificados en factores internos $\mathrm{y}$ externos. Los factores internos asociados al alumno son: el tiempo adicional que el estudiante dedica al estudio, la elección de la carrera deseada, el tiempo dedicado a otras actividades personales, entre otros. Los factores externos son: ingreso económico familiar, calidad de la enseñanza, calidad de la infraestructura de la universidad, entre otros. Este cuestionario incluye preguntas sobre la relación con los académicos, actitud de los docentes, percepción que tiene el alumno de los profesores tanto en su desempeño como en su estrategia de enseñanza, el entorno del alumno en las instalaciones de la universidad incluyendo el salón de clase, laboratorios, biblioteca, infraestructura informática (Salagre, D. J., \& Serrano, S. O. 2003).

La maestra Brito desarrolló un cuestionario para determinar situaciones de riesgo evaluando: peligro, vulnerabilidad y exposición. Este cuestionario consta de 44 preguntas distribuidas en las categorías: motivos y expectativas académicas; hábitos de vida; economía; salud; familia. El cuestionario se podría aplicar al inicio de cada ciclo de formación.

Los cuestionarios se pueden aplicar de forma anónima, pero si el objetivo es apoyar al grupo y determinar la relación de los factores con el desempeño del alumno, es importante que el tutor identifique a quien pertenecen los cuestionarios guardando absoluta confidencialidad.

\section{Métodos estadísticos}

A los datos obtenidos por medio de las pruebas y cuestionarios se aplicarían métodos matemáticos que permitan un análisis para obtener información cuantitativa y objetiva. De los métodos se seleccionó Estadística Descriptiva (Closas, A., Arriola, E. A., Zening, C. I. K., Amarilla, M. R., \& Jovanovich, E. C. 2013). La Estadística Descriptiva proporcionará información importante de la población encuestada como grupo.
Posteriormente se realizaría un Análisis de Regresión Lineal (Jiménez, M. V. G., Izquierdo, J. M. A., \& Blanco, A. J. 2000) para cuantificar la correlación que existe entre alguno de los parámetros y el promedio global del alumno, esto es, se cuantifica la influencia del parámetro en el desempeño académico del alumno y del grupo.

\section{Resultados}

Los tests y cuestionarios se aplicaron a alumnos de un grupo del PE Ingeniería en Redes y Telecomunicaciones como programa piloto. Con referencia al cuestionario que evalúa y mide las factores internos y externos en el desempeño académico se tienen los siguientes resultados de la Estadística Descriptiva: el grupo es de 20 estudiantes del 4o cuatrimestre de la carrera de Ingeniería en Redes y Telecomunicaciones. La edad de los encuestados es entre 19 y 22 años. El $75 \%$ son del género masculino y el $25 \%$ corresponde al género femenino.

El $90 \%$ de los que respondieron el cuestionario no reprobaron ninguna asignatura durante el nivel medio superior, mientras que en la universidad el $30 \%$ reprobó por lo menos una asignatura. Los alumnos mencionaron estar $65 \%$ seguros de haber elegido la carrera, y a su vez, están $66 \%$ satisfechos con ella. Respecto al entendimiento de los temas vistos en clase, los entrevistados afirman comprender los temas en un $67 \%$ y consideran que la calidad de enseñanza de los profesores es del 79\%. Se realizó un análisis de correlación lineal de Pearson, y se obtuvo la relación entre algunas variables internas o externas a los alumnos.

Los alumnos de mayor edad tienden a comprender mejor las clases $(r=0.488)$; se observa una relación de $\mathrm{r}=0.463$, entre el tamaño de las familias y la cantidad de asignaturas reprobadas; asimismo, se observa que los alumnos que dedican tiempo extra a estudiar los temas vistos en clase, obtienen mejores resultados en sus evaluaciones, estas dos variables presentan un grado de correlación de $r=0.490$. Se encontró una relación interesante entre el tiempo de esparcimiento de los alumnos y sus logros académicos, con un coeficiente de correlación $r=0.623$. 
Finalmente, se puede apreciar una correlación negativa entre la cantidad de asignaturas reprobadas en sus estudios de nivel medio superior y el ingreso familiar: a mayor ingreso económico familiar, se presenta un menor rendimiento académico de los estudiantes, presentando un coeficiente de correlación de Pearson $r=-0.417$.

Con referencia al cuestionario de identificación de situaciones de riesgo, se aplicó al mismo grupo por lo que la caracterización obtenida por la estadística descriptiva, se mantiene. La maestra identificó algunos alumnos en situación de riesgo por lo que posterior a una charla para sensibilizar al grupo y regresarles lo que ella detectó a nivel colectivo, pidió poder charlar de manera individual con cada uno de ellos. Algunos alumnos por cuestiones de tiempo y trabajo no acudieron a la entrevista.

Los resultados de HN, RA, RM presentan calificaciones inferiores al $50 \%$, es de esperarse que, al aplicarlos de nuevo, después de dos ciclos de formación completos, estos valores se incrementen significativamente. También se realizó un análisis entre los cuestionarios y las pruebas encontrando dependencia entre las situaciones de riesgo, los factores internos y externos.

De los resultados anteriores, se podría intervenir, como se mencionó, con entrevistas personales por parte de profesionales en psicología o salud. También se podrían realizar charlas referentes a nutrición, uso adecuado del tiempo, economía, deportes, talleres para fomentar la lectura, ética y talleres con los padres de familia.

Como resultado adicional, se propone una estructura sencilla del centro de apoyo que se muestra en la figura 3 .

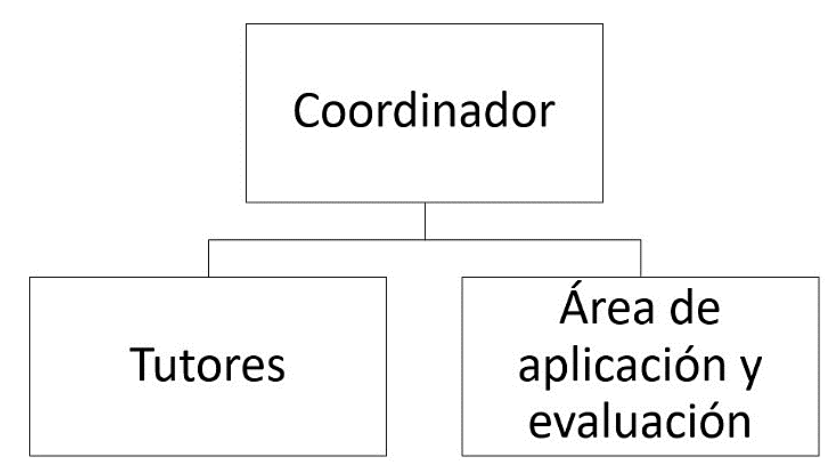

Figura 3 Estructura del CIAACI
El coordinador puede ser el director del PE o alguno de los docentes. Los tutores serían los docentes que forman parte del PE. El área de aplicación podrían ser los mismos docentes, así como profesionales de las áreas de la salud, psicología, deporte, cultura. Esta última área en conjunto con el coordinador y los tutores se podrían elaborar los programas de intervención.

\section{Conclusiones}

Con referencia a los objetivos de este trabajo, se puede concluir que el modelo de flujo de trabajo presentado en la figura 1 proporciona los resultados esperados al organizar a los docentes en su rol de tutores y ayudar a la coordinación entre ellos. Al menos en nuestra universidad, esta situación no es sencilla dada la carga de trabajo, así como las áreas de especialidad de cada docente.

Aun cuando se aplicó sólo en un grupo del PE, al conversar con los otros docentes mostraron interés, así como el director del PE. En la carga de trabajo se incluyen horas de tutoría y asesoría por lo que en ese tiempo se podrían aplicar los cuestionarios y las pruebas e implementar las actividades de intervención y de apoyo individual. Con respecto a las pruebas y cuestionarios, los alumnos los tomaron con cierto recelo al principio, pero al explicarles el objetivo, participaron con menos resistencia. Algunos alumnos no acudieron a la entrevista personal, un poco por cuestiones de horario, otros por resistencia, pero al menos están conscientes de que hay áreas de oportunidad que pueden abordar. Un error que se cometió en este aspecto es que se identificaron públicamente aquellas personas a las que se les invitaba a la entrevista personal. Ello ocasionó en ellos malestar. Se propone realizar la entrevista a todos los alumnos.

En principio no se puede concluir del análisis de los datos si las UA de las asignaturas cursadas hasta ese cuatrimestre han influido positivamente en sus HN, RA y RM por lo que se recomienda aplicar las pruebas al finalizar el segundo ciclo de formación, al final del sexto cuatrimestre o inicio del séptimo. Se podría aplicar también al final del noveno y revisar si las asignaturas de la carrera han aportado positivamente. 
Las situaciones de riesgo cada alumno las puede valorar y al estar conscientes de ello podrían asistir a ayuda profesional o si se suscita alguna situación en el aula, a sugerencia del tutor, canalizarlo a psicología. De los factores internos-externos que pueden influir en el desempeño académico se observó algunas situaciones que podrían ser modificadas para una mejora. Definitivamente este proyecto sólo podría realizarse contando con el apoyo de las autoridades de la institución.

\section{Referencias}

Argudín, Y. (2011) Educación basada en competencias. Educar: revista de educación/nueva época, 16, 1-29

Chávez María Aurora, Ángel S. Pérez, Reynaldo Alanís, "Habilidades Adquiridas al Estudiar una Carrera en la Fac. de Ciencias Físico Matemáticas de la UANL", VIII Congreso Nacional de Tecnologías en la Educación ConTe 2019, 23 al 25 de octubre 2019, Facultad de Ciencias de la Computación, BUAP.

Closas, A., Arriola, E. A., Zening, C. I. K., Amarilla, M. R., \& Jovanovich, E. C. (2013). Análisis multivariante, conceptos y aplicaciones en Psicología Educativa y Psicometría. Enfoques: revista de la Universidad Adventista del Plata, 25(1), 65-92.

Espinosa García, J., \& Román Galán, T. (1991). Actitudes hacia la ciencia y asignaturas pendientes: dos factores que afectan al rendimiento en ciencias. Enseñanza de las Ciencias, 9(2), 151-154.

Jiménez, M. V. G., Izquierdo, J. M. A., \& Blanco, A. J. (2000). La predicción del rendimiento académico: regresión lineal versus regresión logística. Psicothema, 12(Su2), 248525.

Kaplan, R. M., \& Saccuzzo, D. P. (2006). Pruebas psicológicas: principios, aplicaciones y temas. Thomson.

Salagre, D. J., \& Serrano, S. O. (2003). Determinación de los factores que afectan al rendimiento académico en la educación superior. XII Jornadas de la Asociación de Economía de la Educación.
Tobón, S. (2008). La formación basada en competencias en la educación superior: el enfoque complejo. México: Universidad Autónoma de Guadalajara, 5. 